\title{
DYNAMIC PORTFOLIO ALLOCATION, THE DUAL THEORY OF CHOICE AND PROBABILITY DISTORTION FUNCTIONS
}

\author{
BY \\ Mahmoud Hamada, Michael Sherris and John van der Hoek
}

\begin{abstract}
Standard optimal portfolio choice models assume that investors maximise the expected utility of their future outcomes. However, behaviour which is inconsistent with the expected utility theory has often been observed.

In a discrete time setting, we provide a formal treatment of risk measures based on distortion functions that are consistent with Yaari's dual (non-expected utility) theory of choice (1987), and set out a general layout for portfolio optimisation in this non-expected utility framework using the risk neutral computational approach.

As an application, we consider two particular risk measures. The first one is based on the PH-transform and treats the upside and downside of the risk differently. The second one, introduced by Wang (2000) uses a probability distortion operator based on the cumulative normal distribution function. Both risk measures rank-order prospects and apply a distortion function to the entire vector of probabilities.
\end{abstract}

\section{KEYWORDS}

Portfolio allocation, dual theory, probability distortion, equilibrium pricing.

\section{INTRODUCTION}

This paper considers the dynamic optimal consumption and portfolio selection problem, in a discrete-time setting and using a non-expected utility setting. The majority of portfolio choice models assume that preferences are represented by a von Neuman-Morgenstern utility function and individuals choose among risky alternatives so as to maximise the expectation of the utility of possible outcomes. Although the expected utility model has long been the standard for choice under uncertainty, questions have been raised concerning its validity, and behavior patterns which are systematic, yet inconsistent with expected utility theory have often been observed as in the Allais paradox (1953 [1]) and Kahneman \& Tversky (1979 [11]). Fishburn (1988 [6]) surveys the reasons why the 
expected utility hypothesis fails. Camerer (1989 [3]) carries out empirical tests of several generalized models of utility theory. Yaari (1987 [24]) developed a dual theory of choice under risk where the roles of probabilities and payments are interchanged, so the wealth utility function is replaced by a probability distortion function. Some of the expected utility related paradoxes are resolved in the dual theory. The rank dependent utility model introduced by Quiggin $(1982,[15])$ can be viewed as an extension of both the expected utility and the dual utility models where both the cumulative distribution function and the outcomes are distorted. The idea of the rank dependent utility model is to rankorder prospects and apply a distortion function (called weighting function by Quiggin [15]) to the entire vector of probabilities and the utility function to the outcomes.

Recently, there has been development in a non-expected utility framework. Wang (1995 [20], 1996 [19]) proposes calculating insurance premiums by applying the proportional hazards transform to the decumulative distribution function, thereby introducing a new risk measure. This new measure turns out to be consistent with Yaari's dual theory of choice. Wang (2000 [22]) also uses a different class of distortion operators to recover the Black-Scholes formula. Van der Hoek and Sherris (2001 [18]) introduce a new class of risk measures for asset allocation which is based on the distortion function approach to insurance risk.

Empirically, there is evidence to support non-expected utility model. Indeed, Bufman and Leiderman (1990 [2]) use Israeli data between 1978 and 1986 to test an intertemporal consumption-investment model introduced by Epstein and Zin (1989 [5]) that uses Kreps-Porteus (1978 [13]) non-expected utility preferences. They find evidence to reject the expected utility model and accept the non-expected utility one. Their results differ from those of Epstein and Zin (1989b [4]) and Giovannini and Jorion (1989 [7]) who took data from the tranquil postwar US economy. This suggests that a non-expected utility model may perform better in a volatile economy. The results of the empirical tests of the same model using French data from 1960 to 1994 conducted by Koskievic (1999 [12]) support those of Bufman and Leiderman (1990 [2]).

This paper is organised as follows. In section 2, we present the concept of risk aversion for non-expected utility and illustrate the idea of using a distortion function to price risk. New objective for asset allocation is set in non-expected utility framework. Section 3 provides a formal treatment of risk measures based on probability distortion. New class of risk measures for portfolio selection based on the proportional hazards transform, proposed by van der Hoek and Sherris (2001 [18]) is then reviewed and extended to the multinomial case. This is the first step in setting out a general scheme for dynamic asset allocation when the risk measure is based on a distortion function. Some other properties, useful for the optimisation, are developed along the way. In section 4 we derive a dual utility theory equilibrium pricing formula for market securities and propose to solve the optimal portfolio problem using the risk neutral computational approach when the investor behaviour is modeled by this new class 
of risk measures. In section 5, we extend the previous framework from single period to multi-period. In section 6, some numerical examples for asset allocation are provided. The conclusion highlights some further developments.

\section{Non-Expected Utility Theory}

\subsection{Risk aversion in utility theory and its dual}

Decision makers with a von Neumann-Morgenstern utility function are said to be risk averse if they prefer to have the expected value of a gamble rather than facing the gamble itself, i.e. For all gambles $X$ with $\mathbb{E}(X)=0$ and positive variance, and for a level of wealth $W, U(W)>E U(W+X)$.

It can be proved (see Ingersoll [10]) that decision makers are risk averse if and only if their von Neumann-Morgenstern utility function of wealth is strictly concave. Moreover, the level of risk aversion is measured by the degree of concavity of the utility function. Locally, this is determined by Arrow-Pratt's absolute risk aversion index: $A(W)=\frac{u^{\prime \prime}(w)}{u^{\prime}(w)}$. The larger the index, the more riskaverse the agent.

To induce a risk-averse individual to undertake a fair gamble, a compensatory risk premium $\Pi_{c}(X)$ has to be offered. Or dually, to avoid a present gamble, a risk averse individual would be willing to pay an insurance risk premium $\Pi_{i}(X)$. These risk premiums are depicted as follows:

$$
\begin{aligned}
\mathbb{E}\left[U\left(W+\Pi_{c}(X)+X\right)\right] & =U(W) \\
\mathbb{E}[U(W+X)] & =U\left(W-\Pi_{i}(X)\right)
\end{aligned}
$$

The amount $W-\Pi_{i}(X)$ is the amount which, when received with certainty, is considered by the individual as good as $W+X$. It is called the certainty equivalent of the gamble $W+X$.

In the expected utility theory, suppose that an individual must choose among lotteries with at most $n$ outcomes $x_{1}, x_{2}, \ldots, x_{n}$, with respective probabilities $p_{1}$, $p_{2}, \ldots, p_{n}$, then there exists a utility function $U$ such that this individual's choice criterion is to maximise

$$
\mathbb{E}[U(X)]=\sum_{i=1}^{n} p_{i} U\left(x_{i}\right)=\int_{\Omega} u(X(\omega)) d P(\omega)
$$

Note that this objective function is linear in probabilities and distorts the payoffs.

In the dual theory of choice introduced by Yaari [24], the certainty equivalent to $X \geq 0$ is defined as:

$$
\Pi(X)=\int_{0}^{\infty} g\left(S_{X}(t)\right) d t
$$


where $g$ is a "dual utility" or a distortion function (continuous and non-decreasing) $g:[0,1] \rightarrow[0,1]$ with $g(0)=0$ and $g(1)=1$, applied to the probability decumulative distribution:

$$
S_{X}(t)=\operatorname{Pr}[X>t]
$$

This general form of $\Pi(X)$ is valid for continuous and discrete time cases, where the integral sign will be a summation sign in a discrete case, and the appropriate formula is developed later. If $X$ is a non-negative random variable representing a loss amount then $\Pi(X)$ is the certainty equivalent of the risk $X$. In the dual theory, given a choice among risky prospects, the agent would prefer risks having the greatest certainty equivalent.

It can be proved (see Yaari [24]) that the investor is risk averse if and only if $g$ is convex. An intuitive interpretation of this property follows in the case when $g$ is differentiable:

$$
\Pi(X)=\int_{0}^{\infty} g\left(S_{X}(t)\right) d t=\int_{0}^{\infty} \operatorname{tg}^{\prime}\left(S_{X}(t)\right) d F_{X}(t)
$$

assuming that $\operatorname{tg}\left[S_{X}(t)\right] \rightarrow 0$ as $t \rightarrow \infty$. Recall that:

$$
\mathbb{E}[X]=\int_{0}^{\infty} t d F_{X}(t)
$$

Comparing $\Pi(X)$ to $\mathbb{E}[X], \Pi(X)$ can be thought of as a corrected mean of $X$ where the payment $t$ receives a weight $g^{\prime}\left(S_{X}(t)\right) \geq 0$. Note that these weights sum up to 1, i.e. $\int g^{\prime}\left(S_{X}(t)\right) d F_{X}(t)=\int \frac{d}{d t}\left[-g\left(S_{X}(t)\right)\right] d t=g(1)-g(0)=1$.

If $g$ is convex, then

$$
t_{1}>t_{2} \Rightarrow S_{X}\left(t_{1}\right)<S_{X}\left(t_{2}\right) \Rightarrow g^{\prime}\left(S_{X}\left(t_{1}\right)\right)<g^{\prime}\left(S_{X}\left(t_{2}\right)\right)
$$

Therefore, the weight assigned to a high outcome is less than the weight assigned to a low outcome. Hence, by distorting the probabilities with a convex function, agents behave pessimistically, in the sense that they assign high probability to bad outcomes and low probability to good outcomes.

The comparison of risk aversion in this framework is naturally based on the convexity of the function $g$ representing the agent's preference function. The more convex the function $g$, the more risk averse the agent. The dual Arrow-Pratt risk aversion would be in this case $\frac{g^{\prime \prime}(p)}{g^{\prime}(p)}$ for $0<p<1$, as defined in Yarri (1986 [23]). In the sense of Ross (1981 [17]), agents are strongly more risk averse, if they require a larger compensation for any mean preserving spread in their prospects, even if the initial situation is not one of perfect certainty. Risk aversion measurement in the sense of Yaari (1986 [23]) and Ross (1981 [17]) are discussed in Röel (1985 [16]).

To sum up, while risk aversion in utility theory is measured by the utility function, in the dual theory, it is measured by the probability distortion function. 
The choice of the distortion function $g$ determines the properties of the certainty equivalent.

In the literature, Wang (1996 [20]) proposes a general class of distortion operators to use in pricing insurance premiums. When the distortion function is a power function, i.e., $g(x)=x^{r}$, the mapping

$$
S_{X}(t) \rightarrow g\left(S_{X}(t)\right)
$$

is called the $\mathrm{PH}$-transform. Applications and implementation of the PH-transform in insurance is discussed in Wang (1998 [21]). Although the PH-transform enjoys desirable properties in insurance pricing, it cannot be applied to assets and liabilities simultaneously. Wang (2000 [22]) proposes another class of distortion operators

$$
g_{\alpha}(p)=\Phi\left[\Phi^{-1}(p)+\alpha\right]
$$

where $\Phi(p)=\int_{-\infty}^{p} \frac{1}{\sqrt{2 \pi}} e^{-\frac{x^{2}}{2}} d x$ is the standard normal cumulative distribution function and shows how the mean of the distorted decumulative distribution can be used as another alternative to the risk-neutral valuation in asset pricing. Van der Hoek and Sherris (2001 [18]) introduced another framework for pricing asset and liabilities, based on distortion of the probability distribution. They use two different distortion operators, $g$ and $h$ to allow a different pricing of the upside and downside of the risk. The specification of $g$ and $h$ is not given, thereby allowing for a general pricing framework. In the following, we shall consider the certainty equivalent in discrete-time, then overview the risk measure introduced by van der Hoek and Sherris (2001 [18]), develop new properties which are useful for optimisation, and use these results to solve the optimal portfolio problem.

\subsection{New Objective for Asset Allocation}

In multi-period asset allocation, investors are faced with a series of decisions where at the beginning of each period, they have to choose the optimal amount of consumption and investment. The optimal consumption level $C_{t}$ at time $t$ is a risky prospect. Formally, $C_{t}, t=0, \ldots, T$ is a non-negative and bounded random variable defined on some probability space. By virtue of Theorem 2 in Yaari ([23]), the scheme $\left(C_{0}, C_{1}, \ldots, C_{T}\right)$ is preferred to $\left(C_{0}^{\prime}, C_{1}^{\prime}, \ldots, C_{T}^{\prime}\right)$ if and only if there exists an increasing continuous function $U: R_{+}^{n} \rightarrow R_{+}$such that:

$$
U\left(H\left(C_{0}\right), H\left(C_{1}\right), \ldots, H\left(C_{T}\right)\right) \geq U\left(H\left(C_{0}^{\prime}\right), H\left(C_{1}^{\prime}\right), \ldots, H\left(C_{T}^{\prime}\right)\right)
$$

The investor chooses the consumption stream that maximises an increasing function of the certainty equivalent of consumption at each period. 
In what follows, we choose

$$
\begin{gathered}
U: R_{+}^{n} \rightarrow R_{+} \\
\left(x_{1}, x_{2}, \ldots, x_{n}\right) \mapsto \sum \beta^{i} x_{i}
\end{gathered}
$$

where $0<\beta \leq 1$ is the time preference factor. The consumption-investment problem can be reformulated as follows:

$$
\begin{cases}\underset{C_{0}, C_{1}, \ldots, C_{T}}{\operatorname{Max}} & \sum_{t=0}^{T} \beta^{t} H\left(C_{t}\right) \\ \text { s.t. } & W_{0}=\sum_{t=0}^{T} \mathbb{E}_{\mathbf{Q}}\left[\frac{C_{t}}{(1+r)^{t}}\right] \\ & C_{t} \geq 0 \quad \forall t \in[0, T]\end{cases}
$$

where $W_{0}$ is initial wealth and $r$ is a constant interest rate, that can be extended to be varying with time and states of nature. $\mathbf{Q}$ is the risk-neutral probability measure under which the underlying security process is martingale. The objective function is not as tractable as in the expected utility theory. This is because it depends on the order of consumption. In the following section, we develop an expression for the discrete time case.

\section{Asset Allocation in Single-Period}

\subsection{Dual Theory Equilibrium Pricing}

In the dual utility theory the consumer-investor problem for a single-period using the rank-ordered optimisation framework is

$$
\begin{cases}\max _{C_{0}, C_{1}} & C_{0}+\beta H\left(C_{1}\right) \\ \text { subject to } & C_{0}, C_{1} \geq 0 \\ \text { and } & W_{0}=C_{0}+\frac{1}{1+r} \mathbb{E}_{\mathbf{Q}}\left[C_{1}\right]\end{cases}
$$

where $C_{0}$ and $C_{1}$ are consumption at time 0 and 1 respectively, $W_{0}$ is initial wealth, $\beta$ is a time discount factor and $r$ is a constant interest rate, that can be extended to be varying with time and states of nature. $\mathbf{Q}$ is the risk-neutral probability measure under which the underlying security process is martingale.

Suppose that $C_{0}^{*}$ and $C_{1}^{*}$ are solution to (3.1). Perturb the consumption $C_{0}^{*}$ in such a way that the consumer consumes less than $C_{0}^{*}$ in order to invest in a security $j$ whose price at time 0 is $x_{j}$. This translates to 


$$
\begin{aligned}
& t=0: C_{0}^{* \prime}=C_{0}^{*}-\xi x_{j} \\
& t=1: C_{1}^{* \prime}=C_{1}^{*}+\xi X_{j}
\end{aligned}
$$

where $\xi$ is the fraction invested in the security $j$ and $X_{j}$ is the security $j$ price at time 1: The objective is

$$
\begin{aligned}
\text { Obj } & =C_{0}^{*}-\xi x_{j}+\beta H\left(C_{1}^{*}+\xi X_{j}\right) \\
& =C_{0}^{*}-\xi x_{j}+\beta \hat{\mathbb{E}}_{h}\left[C_{1}^{*}+\xi X_{j}\right]
\end{aligned}
$$

where

$$
\hat{\mathbb{E}}_{h}\left[C_{1}^{*}+\xi X_{j}\right]=\sum_{\Omega} \mathbf{P}_{\xi}^{h}(\omega)\left(C_{1}^{*}(\omega)+\xi X_{j}(\omega)\right)
$$

and

$$
\begin{gathered}
\mathbf{P}_{\xi}^{h}(\omega)=h\left(\mathbf{P}\left[C_{1}^{*}+\xi X_{j} \geq C_{1}^{*}(\omega)+\xi X_{j}(\omega)\right]\right)- \\
h\left(\mathrm{P}\left[C_{1}^{*}+\xi X_{j}>C_{1}^{*}(\omega)+\xi X_{j}(\omega)\right]\right)
\end{gathered}
$$

Note that this is not an expectation since the weights $\mathbf{P}_{\xi}^{h}(\omega)$ depend on $X$ and the operator $\hat{\mathbb{E}}_{h}$ is not linear.

We know that $O b j$ is maximized for $\xi=0$ (since $C_{0}^{*}$ and $C_{1}^{*}$ are optimal). If $O b j$ is concave in $\xi$ and differentiable then it is maximized when the derivative $\frac{\partial O b j}{\partial \xi}$ is zero.

$$
\frac{\partial O b j}{\partial \xi}=-x_{j}+\beta \sum_{\Omega} \frac{\partial P_{\xi}^{h}}{\partial \xi}(\omega)\left(C_{1}^{*}(\omega)+\xi X_{j}(\omega)\right)+\beta \sum_{\Omega} \mathbf{P}_{\xi}^{h}(\omega) X_{j}(\omega)
$$

Now, $\left.\frac{\partial O b j}{\partial \xi}\right|_{\xi=0}=0$ implies

$$
\begin{aligned}
x_{j} & =\beta \sum_{\Omega} \mathbf{P}_{0}^{h}(\omega) X_{j}(\omega)+\beta \sum_{\Omega} \mathbf{P}_{0}^{h{ }^{\prime}}(\omega) C_{1}^{*}(\omega) \\
& =\beta H\left(X_{j}\right)+\beta \sum_{\Omega} \mathbf{P}_{0}^{h^{\prime}}(\omega) C_{1}^{*}(\omega)
\end{aligned}
$$

This gives a relationship between the price of the security $j$ at time 0 and time 1 : If $\mathbf{P}_{0}^{h^{\prime}}=0$, then we have the pricing result suggested by Wang (2000 [22])

$$
x_{j}=\beta H\left(X_{j}\right)
$$

With these ingredients, we propose to study some classes of risk measures based on distortion functions and consider the application to asset allocation. 


\subsection{Application: Van der Hoek and Sherris class of risk measures}

In their paper, van der Hoek and Sherris (2001 [18]) define the certainty equivalent of a random variable $X$ by:

$$
\begin{aligned}
H(X) & \equiv H_{\alpha, g, h}(X)=\alpha+H_{h}\left((X-\alpha)^{+}\right)-H_{g}\left((\alpha-X)^{+}\right) \\
& =\alpha+\int_{0}^{\infty} h\left\{\operatorname{Pr}\left[(X-\alpha)^{+}>t\right]\right\} d t-\int_{0}^{\infty} g\left\{\operatorname{Pr}\left[(\alpha-X)^{+}>t\right]\right\} d t
\end{aligned}
$$

where $\alpha$ is a real constant, and $h$ is a convex and increasing function on $[0,1]$ with $h(0)=0$ and $h(1)=1$, and $g$ is a concave and increasing function on $[0,1]$ with $g(0)=$ and $g(1)=1$. The convexity and concavity of $h$ and $g$ ensures the concavity of $H_{\alpha, g, h}(X)$ which is an appealing property in portfolio optimisation.

Definition 1. The functions $g$ and $h$ are said to be conjugate if and only if: $h(x)=$ $1-g(1-x) \forall x \in[0,1]$

In what follows, we provide an expression of $H(X)$ in the discrete-time case.

Proposition 1 (Order assumption). If $X$ is a multinomial discrete random variable taking the values $\left(x_{1}, x_{2}, \ldots, x_{n}\right)$ such that $x_{1}<x_{2}<\ldots<x_{n}$, with probabilities $\left(p_{1}\right.$, $\left.p_{2}, \ldots, p_{n}\right)$, then,

$$
H(X)=\alpha+\sum_{i=1}^{n}\left[h_{i-1}^{p}-h_{i}^{p}\right]\left(x_{i}-a\right)^{+}-\left[g_{i}^{p}-g_{i-1}^{p}\right]\left(\alpha-x_{i}\right)^{+}
$$

where

$$
h_{i}^{p}=h\left(1-\sum_{k=1}^{i} p_{k}\right) \text { and } g_{i}^{p}=g\left(\sum_{k=1}^{i} p_{k}\right)
$$

Proof. The idea of the proof is the same as in the proof of Theorem (Certainty equivalent). A detailed proof is provided in the appendix.

In its general form, $H(X)$ is a piecewise linear function, so it is not differentiable. However, when $x_{1}, x_{2}, \ldots, x_{n}$ and $\alpha$ can be ordered, then $H(X)$ has a simple differentiable form given by the corollary (2) in the appendix. More useful properties of $H$ are detailed in the appendix.

In the case of a one-period model with one risky security and one riskless asset, there are 5 unknown variables: $C_{0}$, the consumption at time $0, C_{1}^{u}$ and $C_{1}^{d}$, the consumption at time 1 for the up and down states and $H_{0}$ and $H_{1}$ the investment positions in the safe and the risky asset respectively. To solve this problem, we start from the budget constraints that involve both consumption and investment strategies and express all the variables in terms of $C_{1}^{u}$ and $C_{1}^{d}$. We then show how this is equivalent to using the risk-neutral computational 
approach directly, which from the start determines the constraints in terms of $C_{1}^{u}$ and $C_{1}^{d}$.

The consumption-investment problem is

$$
\begin{cases}\max _{(C, H)} & H\left(C_{0}\right)+\beta H\left(C_{1}\right) \\ \text { subject to } & C_{0}, C_{1} \geq 0 \\ \text { and } & \text { budget constraints }\end{cases}
$$

where the budget constraints are

$$
\begin{aligned}
& W_{0}-C_{0}=H_{0} B_{0}+H_{1} S_{0} \\
& W_{1}-C_{1}=H_{0} B_{1}+H_{1} S_{1}
\end{aligned}
$$

where $B_{0}=1$. We can rewrite all the variables in terms of $C_{1}^{u}$ and $C_{1}^{d}$, (see figure (1)) which become the control variables in problem (3.4).

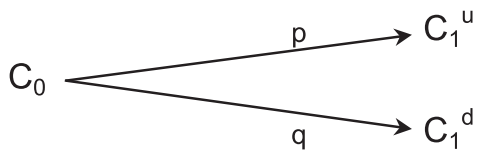

Figure 1. Consumption Nodes in Single-Period Binomial Model.

Equation (3.6) yields two equations

$$
\begin{aligned}
& C_{1}^{u}=H_{0} B_{1}+H_{1} S_{1}^{u} \\
& C_{1}^{d}=H_{0} B_{1}+H_{1} S_{1}^{d}
\end{aligned}
$$

Solving then for $H_{0}$ and $H_{1}$ :

$$
\begin{aligned}
& H_{0}=-\frac{S_{1}^{d}}{B_{1}\left(S_{1}^{u}-S_{1}^{d}\right)} C_{1}^{u}+\frac{S_{1}^{u}}{B_{1}\left(S_{1}^{u}-S_{1}^{d}\right)} C_{1}^{d} \\
& H_{1}=\frac{1}{S_{1}^{u}-S_{1}^{d}} C_{1}^{u}-\frac{1}{S_{1}^{u}-S_{1}^{d}} C_{1}^{d}
\end{aligned}
$$

Using equations (3.5) and (3.7), $C_{0}$ can be expressed in terms of $C_{1}^{u}$ and $C_{1}^{d}$.

$$
C_{0}=W_{0}-b_{1} C_{1}^{u}-b_{2} C_{1}^{d}
$$


where

$$
b_{1}=\frac{B_{1} S_{0}-S_{1}^{d}}{B_{1}\left(S_{1}^{u}-S_{1}^{d}\right)} \quad \text { and } \quad b_{2}=\frac{S_{1}^{u}-B_{1} S_{0}}{B_{1}\left(S_{1}^{u}-S_{1}^{d}\right)}
$$

\section{Interpretation of $b_{1}$ and $b_{2}$ :}

For the choice of $S_{1}^{d}<B_{1} S_{0}<S_{1}^{u}$, it is easy to check that $\mathbf{Q}\left(\omega_{1}\right)=\left(B_{1} S_{0}-S_{1}^{d}\right) /$ $\left(S_{1}^{u}-S_{1}^{d}\right)$ and $\mathbf{Q}\left(\omega_{2}\right)=\left(S_{1}^{u}-B_{1} S_{0}\right) /\left(S_{1}^{u}-S_{1}^{d}\right)$ define a martingale measure for the discounted price $S_{1} / B_{1}$. Therefore

$$
b_{1} C_{1}^{u}+b_{2} C_{1}^{d}=\mathbb{E}_{\mathbf{Q}}\left[\frac{C_{1}}{B_{1}}\right]
$$

And equation (3.8) can be written as

$$
W_{0}=C_{0}+\mathbb{E}_{\mathbf{Q}}\left[\frac{C_{1}}{B_{1}}\right]
$$

The absence of arbitrage condition is equivalent to $\mathbf{Q}\left(\omega_{i}\right) \in(0,1)$ for $i \in\{1,2\}$. Provided that $B_{1}=(1+r) \geq 1$, we also have $b_{i} \equiv \mathbf{Q}\left(\omega_{i}\right) / B_{1} \in(0,1)$.

The problem (3.4) is then equivalent to

$$
\begin{array}{ll}
\max _{(C, H)} & H\left(C_{0}\right)+\beta H\left(C_{1}\right) \\
\text { subject to } & C_{0}, C_{1} \geq 0 \\
& W_{0}=C_{0}+\mathbb{E}_{\mathbf{Q}}\left[C_{1} /(1+r)\right]
\end{array}
$$

This would be the starting point if the optimal portfolio problem (3.4) was formulated within the risk-neutral computational approach.

The constraints of Problem (3.4) are:

$$
\begin{aligned}
& \text { (1) } C_{1}^{u} \geq 0 \text { and } C_{1}^{d} \geq 0 \\
& \text { (2) } W_{0}-C_{0} \geq 0 \Rightarrow b_{1} C_{1}^{u}+b_{2} C_{1}^{d} \geq 0 \text { (using (3.8)) } \\
& \text { (3) } C_{0} \geq 0 \Rightarrow b_{1} C_{1}^{u}+b_{2} C_{1}^{d} \leq W_{0} \text { (using (3.8)) }
\end{aligned}
$$

Solving the Problem - Feasible Region. In this section, we consider the special case when the distortion functions $g$ and $h$ are conjugate, and we solve the problem analytically. The general case of non-conjugate distortion functions is left to the next paragraph dealing with the case of 2 risky assets and 1 riskless security. 
Let us write all the variables in terms of $C_{1}^{u}$ and $C_{1}^{d}$ :

$$
\begin{aligned}
& \text { - } H\left(C_{0}\right)=C_{0}=W_{0}-b_{1} C_{1}^{u}-b_{2} C_{1}^{d} \\
& \text { - } H\left(C_{1}\right)= \begin{cases}h(p) C_{1}^{u}+[1-h(p)] C_{1}^{d} & \text { if } C_{1}^{u} \geq C_{1}^{d} \\
h(q) C_{1}^{d}+[1-h(q)] C_{1}^{u} & \text { if } C_{1}^{u} \leq C_{1}^{d}\end{cases}
\end{aligned}
$$

Therefore, the objective function in the problem (3.9) is equal to $H\left(C_{0}\right)+\beta H\left(C_{1}\right)= \begin{cases}W_{0}+\left\{\beta h(p)-b_{1}\right\} C_{1}^{u}+\left\{\beta(1-h(p))-b_{2}\right\} C_{1}^{d} & \text { if } C_{1}^{u} \geq C_{1}^{d} \\ W_{0}+\left\{\beta(1-h(q))-b_{1}\right\} C_{1}^{u}+\left\{\beta h(q)-b_{2}\right\} C_{1}^{d} & \text { if } C_{1}^{u} \leq C_{1}^{d}\end{cases}$

We consider then the two maximization problems

$$
P_{1}= \begin{cases}\max _{C_{1}^{u}, C_{1}^{d}} & \left\{\beta h(p)-b_{1}\right\} C_{1}^{u}+\left\{\beta(1-h(p))-b_{2}\right\} C_{1}^{d} \\ \text { subject to } & C_{1}^{u} \geq 0, C_{1}^{d} \geq 0 \\ & C_{1}^{u} \geq C_{1}^{d} \\ & b_{1} C_{1}^{u}+b_{2} C_{1}^{d} \leq W_{0}\end{cases}
$$

and

$$
P_{2}= \begin{cases}\max _{C_{1}^{u}, C_{1}^{d}} & \left\{\beta(1-h(q))-b_{1}\right\} C_{1}^{u}+\left\{\beta h(q)-b_{2}\right\} C_{1}^{d} \\ \text { subject to } & C_{1}^{u} \geq 0, C_{1}^{d} \geq 0 \\ & C_{1}^{u} \leq C_{1}^{d} \\ & b_{1} C_{1}^{u}+b_{2} C_{1}^{d} \leq W_{0}\end{cases}
$$

Maximizing (3.4) is equivalent to solving $P_{1}$ and $P_{2}$ and choosing the solution that corresponds to the higher objective value function. The two maximization problems are linear programs that can be solved numerically using any optimization software package. However, since there are only two choice variables, an analytical solution can be given explicitly.

Remark 1. If the probability distribution is such that $\mathbf{P}\left\{\omega_{1}\right\}=\mathbf{P}\left\{\omega_{2}\right\}=\frac{1}{2}$, then $P_{1}$ and $P_{2}$ have the same solution and therefore, it suffices to solve either the problem $P_{1}$ or $P_{2}$. 


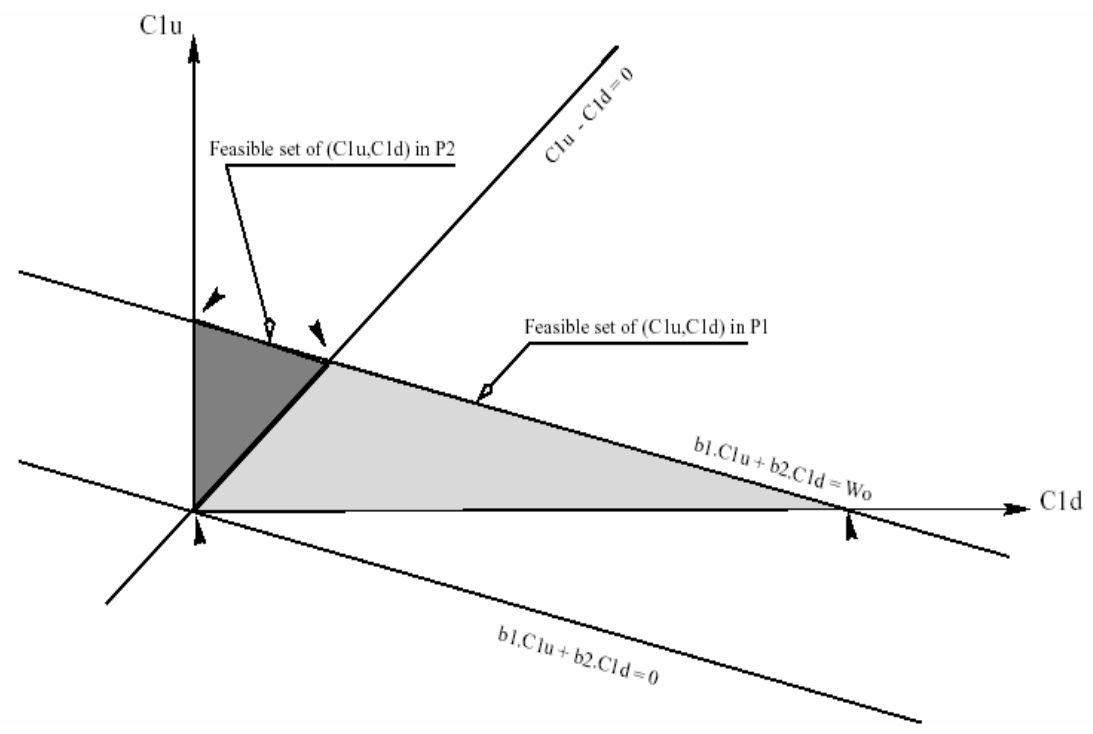

FIGURE 2. Feasible regions for consumption in one-period model.

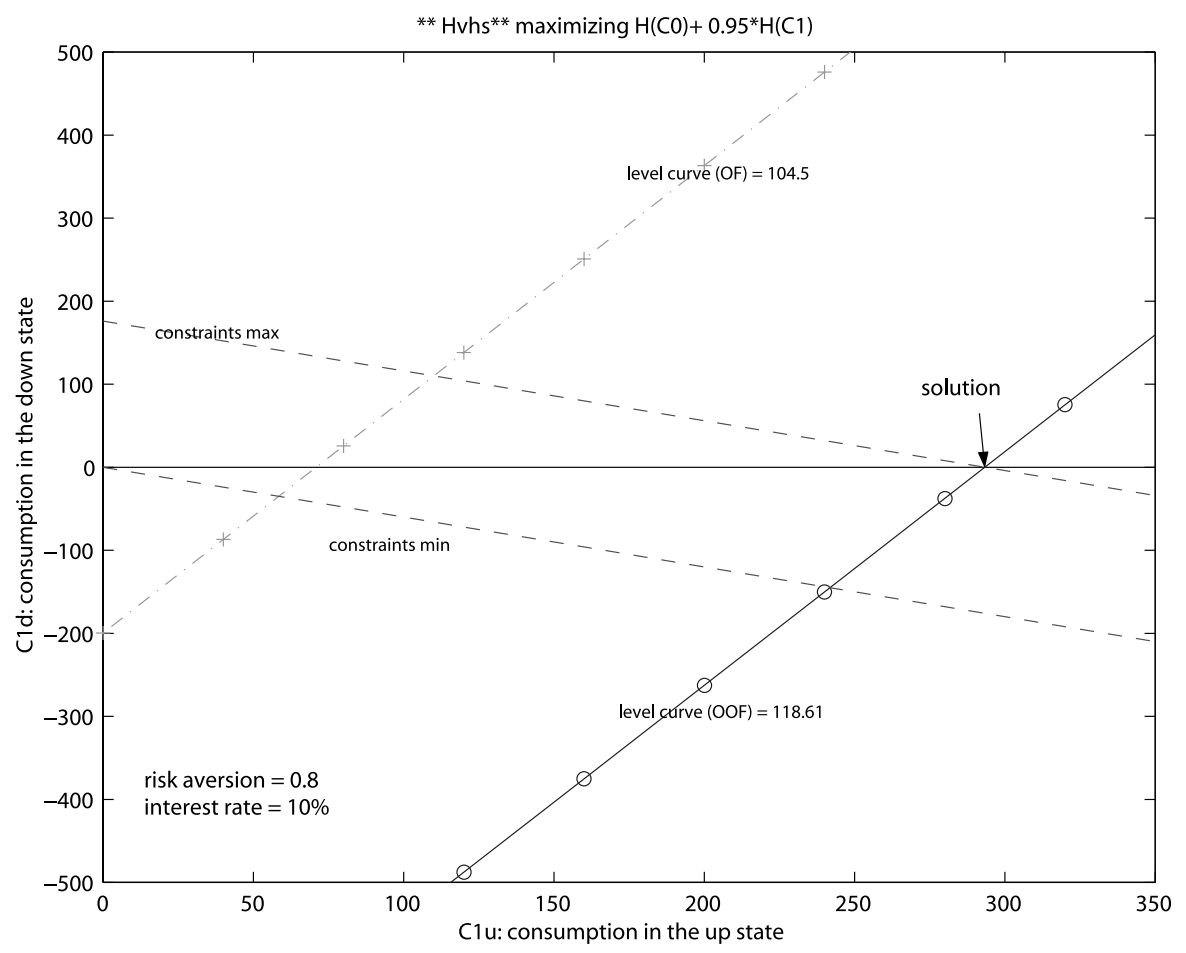

Figure 3. Solution to the Single-Period Portfolio Problem in the Dual Theory. 
Given the constraints, feasible sets of the solution to $P_{1}$ and $P_{2}$ are illustrated in Figure (2). Possible solutions are indicated by the little arrows.

The objective function in the problem (3.10) is linear in $C_{1}^{u}$ and $C_{1}^{d}$. The equation of the line representing the level curve of the objective function at a possible value $z_{1}$ is given by

$$
C_{1}^{d}=\frac{z_{1}}{\beta(1-h(p))-b_{2}}-\frac{\beta h(p)-b_{1}}{\beta(1-h(p))-b_{2}} C_{1}^{u}
$$

There are four possible solutions of the problem corresponding to the summits of the feasible region

$$
(0,0),\left(0, \frac{W_{0}}{b_{2}}\right),\left(\frac{W_{0}}{b_{1}+b_{2}}, \frac{W_{0}}{b_{1}+b_{2}}\right) \text { and }\left(\frac{W_{0}}{b_{1}}, 0\right) .
$$

Figure 3 plots the solution for the case where $\beta=0.95, r=10 \%$ and $\rho=0.8$. Optimal Investment Strategies. Once the optimal consumption rules are obtained, the optimal investment strategies follow from the budget equations (3.7).

- When $\left(C_{1}^{u}, C_{1}^{d}\right)=(0,0)$, then $\left(H_{0}, H_{1}\right)=(0,0)$

- When $\left(C_{1}^{u}, C_{1}^{d}\right)=\left(\frac{W_{0}}{b_{1}}, 0\right)$, then $\left(H_{0}, H_{1}\right)=\left(-W_{0} \frac{S_{1}^{d}}{B_{1} S_{0}-S_{1}^{d}}, W_{0} \frac{B_{1}}{B_{1} S_{0}-S_{1}^{d}}\right)$.

- When $\left(C_{1}^{u}, C_{1}^{d}\right)=\left(\frac{w_{0}}{b_{1}+b_{2}}, \frac{w_{6}}{b_{1}+b_{2}}\right)$, then $\left(H_{0}, H_{1}\right)=\left(W_{0}, 0\right)$. This situation is referred to as "plunging".

- When $\left(C_{1}^{u}, C_{1}^{d}\right)=\left(0, \frac{W_{6}}{b_{2}}\right)$, then $\left(H_{0}, H_{1}\right)=\left(W_{0} \frac{S_{1}^{u}}{S_{1}^{u}-B_{1} S_{0}},-W_{0} \frac{B_{1}}{S_{1}^{u}-B_{1} S_{0}}\right)$.

\section{OPTIMAL PORTFOLIO CHOICE IN A MULTI-PERIOD MODEL}

\subsection{General Set-up}

As discussed in Section 2.2, the consumption-investment problem is:

$$
\begin{cases}\underset{C_{0}, C_{1}, \ldots, C_{T}}{\operatorname{Max}} & \sum_{t=0}^{T} \beta^{t} H\left(C_{t}\right) \\ \text { s.t. } & v=\sum_{t=0}^{T} \mathbb{E}_{\mathbf{Q}}\left[\frac{C_{t}}{(1+r)^{t}}\right] \\ & C_{t} \geq 0 \quad \forall t \in[0, T]\end{cases}
$$

where $v$ is the initial wealth and $r$ is a constant interest rate. 
Problem (4.1) is set up in the discrete-time case. However, it can be solved numerically either with a finite number or a continuum states at each time period.

Let us consider the case when at each time $t, C_{t}$ takes $(2 t+1)$ possible values (recombining trinomial tree), then $C_{t}$ is a vector of $2 t+1$ control variables, i.e.

$$
C_{t}=\left[C_{t,-t}, \ldots, C_{t, 0}, \ldots, C_{t, t}\right] \in \mathbb{R}_{+}^{2 t+1}
$$

For each vector $C_{t}$, there exists a corresponding vector

$$
p_{t}=\left[p_{t,-t}, \ldots, p_{t, 0}, \ldots, p_{t, t}\right] \in[0,1]^{2 t+1}
$$

where $p_{t, i}=P\left[C_{t}=C_{t, i}\right]$.

From Proposition (No order assumption), one way to write the term $H\left(C_{t}\right)$ in the objective function is:

$$
H\left(C_{t}\right)=\sum_{i=-t}^{t} F_{C_{t, i}}\left(p_{t}\right) \cdot C_{t, i}
$$

Where the function

$$
\begin{aligned}
& F_{C_{t, i}}:[0,1]^{2 t+1} \longrightarrow \mathbb{R}_{+} \\
& p_{t}=\left[p_{t,-t}, \ldots, p_{t, 0}, \ldots, p_{t, t}\right] \longmapsto \\
& \left\{\begin{array}{c}
{\left[g\left(\sum_{\left\{C_{t, k} \leq C_{t, i}\right\}} p_{k}\right)-g\left(\sum_{\left\{C_{t, k}<C_{t, i}\right\}} p_{k}\right)\right] I_{\left\{C_{t, i} \leq \alpha\right\}}} \\
+\left[h\left(1-\sum_{\left\{C_{t, k}<C_{t, i}\right\}} p_{k}\right)-h\left(1-\sum_{\left\{C_{t, k} \leq C_{t, i}\right\}} p_{k}\right)\right] I_{\left\{C_{t, i}>\alpha\right\}}
\end{array}\right\}
\end{aligned}
$$

Remark 2. Another formulation is also possible using Proposition (Order assumption), where the control variables are ordered explicitly.

The general expression (4.2) can be significantly simplified in the case when $g$ and $h$ are conjugate functions and the consumption at each time is distributed with the same probability over the states, i.e.

$$
g(x)=1-h(1-x) \quad \forall x \in[0,1]
$$

and

$$
P\left[C_{t}=C_{t, i}\right]=q_{t}=\frac{1}{2 t+1} \quad \forall t \in[1, T], \quad \forall i \in[-t, t]
$$


Then, using Proposition (conjugate), we have

$$
F_{C_{t, i}}\left(p_{t}\right)=g\left(\frac{1}{2 t+1} \#\left\{C_{t, k} \leq C_{t, i}\right\}\right)-g\left(\frac{1}{2 t+1} \#\left\{C_{t, k}<C_{t, i}\right\}\right)
$$

where $\#\left\{C_{t, k} \leq C_{t, i}\right\}$ denotes the number of variables $C_{t, k}, k \in\{-t, \ldots, t\}$, such that $C_{t, k} \leq C_{t, i}$.

In this case, the problem (4.1) can be solved using one of the simplicial algorithms used in rank regression problems. Osborne (2001 [14]) is an excellent reference for solving such types of problems.

\subsection{Application: Wang's class of distortion operators}

Now consider another class of distortion operators introduced by Wang (2000 [22]). Wang shows that applying this distortion operator to a stock price distribution, the risk neutral valuation of stock prices can be recovered in the normal and the lognormal cases. Further investigations, however, should be carried out to check whether this statement is true for any contingent claim, and also when there is no normality assumption on the underlying asset prices. Hamada \& Sherris (2001[8]) provide some insight into this question.

4.2.1. The operator. Let $X$ be a random variable with a decumulative distribution function $S_{X}(x)=P[X>x]$. The expectation of $X$ is alternatively given by:

$$
\mathbb{E}[X]=\int_{-\infty}^{0}\left[S_{X}(x)-1\right] d x+\int_{0}^{\infty} S_{X}(x) d x
$$

Let $\Phi(u)=\int_{-\infty}^{u} \frac{1}{\sqrt{2 \pi}} e^{-\frac{x^{2}}{2}} d x$ be the standard normal cumulative function and $\alpha \in \mathbb{R}$, the distortion operator is defined as:

$$
g_{\alpha}(u)=\Phi\left[\Phi^{-1}(u)+\alpha\right]
$$

for $u$ in $[0,1]$. The risk-adjusted premium of $X$, as defined by Wang (2000) admits the following Choquet representation

$$
H[X, \alpha]=\int_{-\infty}^{0}\left\{g_{\alpha}\left[S_{X}(x)\right]-1\right\} d x+\int_{0}^{\infty} g_{\alpha}\left[S_{X}(x)\right] d x
$$

When $X$ is positive, we have:

$$
H[X, \alpha]=\int_{0}^{\infty} g_{\alpha}\left[S_{X}(t)\right] d t
$$


The risk-adjusted premium is evaluated as in Yaari's dual theory of choice under uncertainty. The tail distribution $S_{X}(t)$ is distorted by the function $g_{\alpha}(p)=$ $\Phi\left[\Phi^{-1}(p)+\alpha\right]$. This operator shifts the $p^{\text {th }}$ quantile of $X$ by a positive or negative value $\alpha$ and reevaluates the normal cumulative probability of the shifted quantile.

If $\alpha>0$, then $g_{\alpha}(p)>p$, if $\alpha<0$, then $g_{\alpha}(p)<p$. Since $g_{\alpha}$ is continuous and $g_{\alpha}(p) \in[0,1]$, then:

$$
\begin{aligned}
& g_{\alpha} \text { is convex if } \alpha<0 \\
& g_{\alpha} \text { is concave if } \alpha>0
\end{aligned}
$$

The investor behaves pessimistically by shifting the quantiles to the left, thereby assigning high probabilities to low outcomes, and behaves optimistically by shifting the quantiles to the right thereby assigning high probabilities to high outcomes. Typically, an insurer has a lower $\alpha$ than a reinsurer when pricing the same risk.

4.2.2. The portfolio problem. In asset allocation, at each time period, the consumption $C_{t}$ is a positive random variable. The investor seeks to maximise the discounted sum of the certainty equivalents of consumption through time, as described by the problem:

$$
\begin{cases}\max _{C} & \sum_{t=0}^{T} \beta^{t} \cdot H\left[C_{t}, \alpha\right] \\ \text { subject to } & \sum_{t=0}^{T} B_{t}^{-1} \cdot \mathbb{E}_{Q}\left[C_{t}\right]=v \\ & C \text { is an adapted process }\end{cases}
$$

where

$$
H\left[C_{t}, \alpha\right]=\int_{0}^{\infty} g_{\alpha}\left(P\left[C_{t}>x\right]\right) d x
$$

If the model consists of a finite number of states at each time period, then $C_{t}$ takes $n_{t}$ possible values $c_{t, 1}, c_{t, 2}, \ldots, c_{t, n_{t}}$ with respective probabilities $p_{t, 1}, p_{t, 2}, \ldots$, $p_{t, n_{t}}$ where $n_{t}$ is the number of states at time $t$. Using Corollary (Certainty equivalent),

$$
H\left[C_{t}, \alpha\right]=\sum_{i=1}^{n_{t}} \frac{\left[g_{\alpha}\left(P\left[C_{t} \geq c_{t, i}\right]\right)-g\left(P\left[C_{t} \geq c_{t, i}\right]\right)\right]}{\#\left\{c_{t, j}: c_{t, j}=c_{t, i}\right\}} c_{t, i}
$$

The probability that $C_{t} \geq c_{t, i}$ is equal to the sum of the probability weights $p_{k}$, such that $c_{t, k} \geq c_{t, i}$, i.e. 


$$
P\left[C_{t} \geq c_{t, i}\right]=\sum_{\left\{k: c_{t, k} \geq c_{t, i}\right\}} p_{t, k}
$$

Hence,

$$
H\left[C_{t}, \alpha\right]=\sum_{i=1}^{n_{t}} \frac{\left[g_{\alpha}\left(\sum_{\left\{k: c_{t, k} \geq c_{t, i}\right\}} p_{t, k}\right)-g\left(\sum_{\left\{k: c_{t, k}>c_{t, i}\right\}} p_{t, k}\right)\right]}{\#\left\{c_{t, j}: c_{t, j}=c_{t, i}\right\}} c_{t, i}
$$

By defining the risk-neutral probability and using the expression above, the description of the problem is complete. This is not a linear program, however it can be solved using an optimisation package. The next paragraph shows how to solve it on a trinomial lattice and provides some results in two periods.

4.2.3. How to compute $H[C, \alpha]$ over a lattice? Fix a time $t$, and consider the distribution of the consumption represented by the vertical nodes $(t, i)_{-t \leq i \leq t}$. At time $t$, the consumption $C_{t}$ takes $2 t+1$ possible values $c_{t, i}$ with probabilities $p_{t, i}=P\left[S=S_{t, i}\right]$; see Figure (4). Hence,

$$
H\left[C_{t}, \alpha\right]=\sum_{i=-t}^{t} \frac{\left[g_{\alpha}\left(\sum_{\left\{k: c_{t, k} \geq c_{t, i}\right\}} p_{t, k}\right)-g_{\alpha}\left(\sum_{\left\{k: c_{t, k}>c_{t, i}\right\}} p_{t, k}\right)\right]}{\#\left\{c_{t, j}: c_{t, j}=c_{t, i}\right\}} c_{t, i}
$$

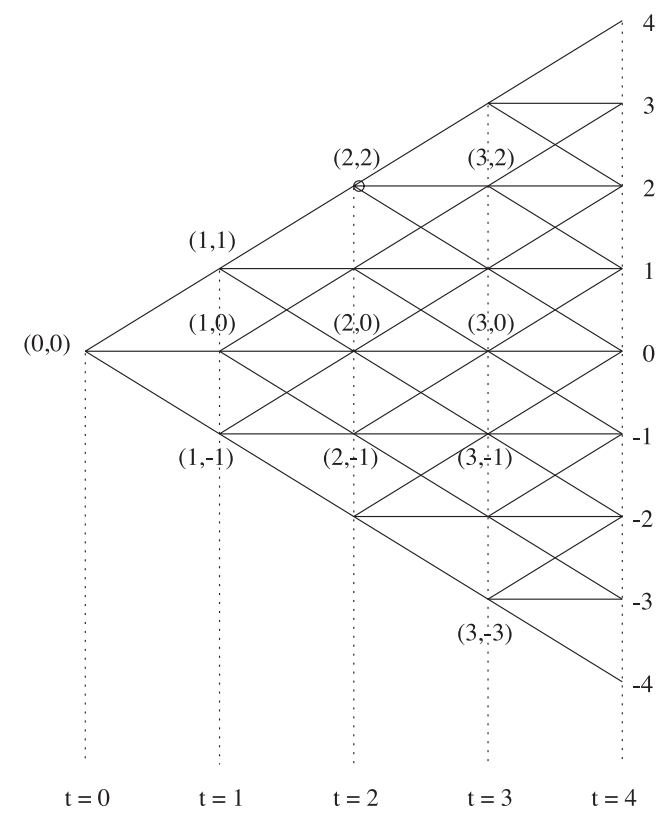

Figure 4. Trinomial lattice encoding. 
The probabilities $p_{t, i}, t \in\{1, \ldots, T\}, i \in\{-t, \ldots, 0, \ldots, t\}$ can be specified as follows:

$$
\begin{aligned}
p_{t, i} & =P\left[S=S_{t, i}\right] \\
& =\sum_{\max (0, i) \leq k \leq\left[\frac{t+i}{2}\right]}\left(\begin{array}{l}
t \\
k
\end{array}\right)\left(\begin{array}{c}
t-k \\
k-i
\end{array}\right) \cdot p_{u}^{k} \cdot p_{d}^{k-i} \cdot\left(1-p_{u}-p_{d}\right)^{t-2 k+i}
\end{aligned}
$$

where $p_{u}$ and $p_{d}$ are respectively the probabilities of up and down jumps and $[x]$ is the integer part of $x$. The expression (4.4) is a generalisation of the probabilities in a binomial model. Likewise, the expression $\mathbb{E}_{Q}\left[C_{t}\right]$ in the constraints can be computed. In effect,

$$
\mathbb{E}_{Q}\left[C_{t}\right]=\sum_{i=-t}^{t} Q_{t, i} \cdot c_{t, i}
$$

where:

$$
\begin{aligned}
Q_{t, i} & =Q\left[S=S_{t, i}\right] \\
& =\sum_{\max (0, i) \leq k \leq\left[\frac{t+i}{2}\right]}\left(\begin{array}{c}
t \\
k
\end{array}\right)\left(\begin{array}{c}
t-k \\
k-i
\end{array}\right) \cdot q_{u}^{k} \cdot q_{d}^{k-i} \cdot\left(1-q_{u}-q_{d}\right)^{t-2 k+i}
\end{aligned}
$$

where $q_{u}$ and $q_{d}$ are respectively the risk-neutral probabilities of up and down jumps.

\section{Numerical Results}

This section provides two numerical examples of portfolio allocation using the classes of distortion operators introduced earlier.

The first example considers Wang's distortion operator. Suppose that there are three dates $t=0,1,2$ and five states of the world. This corresponds to a recombining trinomial lattice. We numerically solve the problem (4.3) for $T=2$. For a loading parameter $\alpha=0.5$, Figure (5) shows the consumption and investment strategies as well as the wealth process for a two period example. The discount factor $\beta=0.9$, the risk-free interest rate $r=10 \%$ and initial wealth $v=\$ 10$.

The jump probabilities are $p_{u}=p_{m}=p_{d}=\frac{1}{3}$ and $q_{u}=\frac{2}{15}, q_{m}=\frac{1}{3}$ and $q_{d}=\frac{8}{15}$. For this choice of risk neutral probabilities, and a correlation coefficient $\rho=0.75$, the means and volatilities of the two risky securities are respectively: mean $_{1}=$ $13.46 \%$, volatility $_{1}=07.07 \%$, and mean $_{2}=15.20 \%$, volatility $_{2}=10.61 \%$.

The intermediate consumption $C$ is null and the consumption is strictly positive at the highest state of the world. However, intermediate positions $n r$, $r 1$ and $r 2$ in the riskless asset, the first security and the second security respectively are nonzero. 


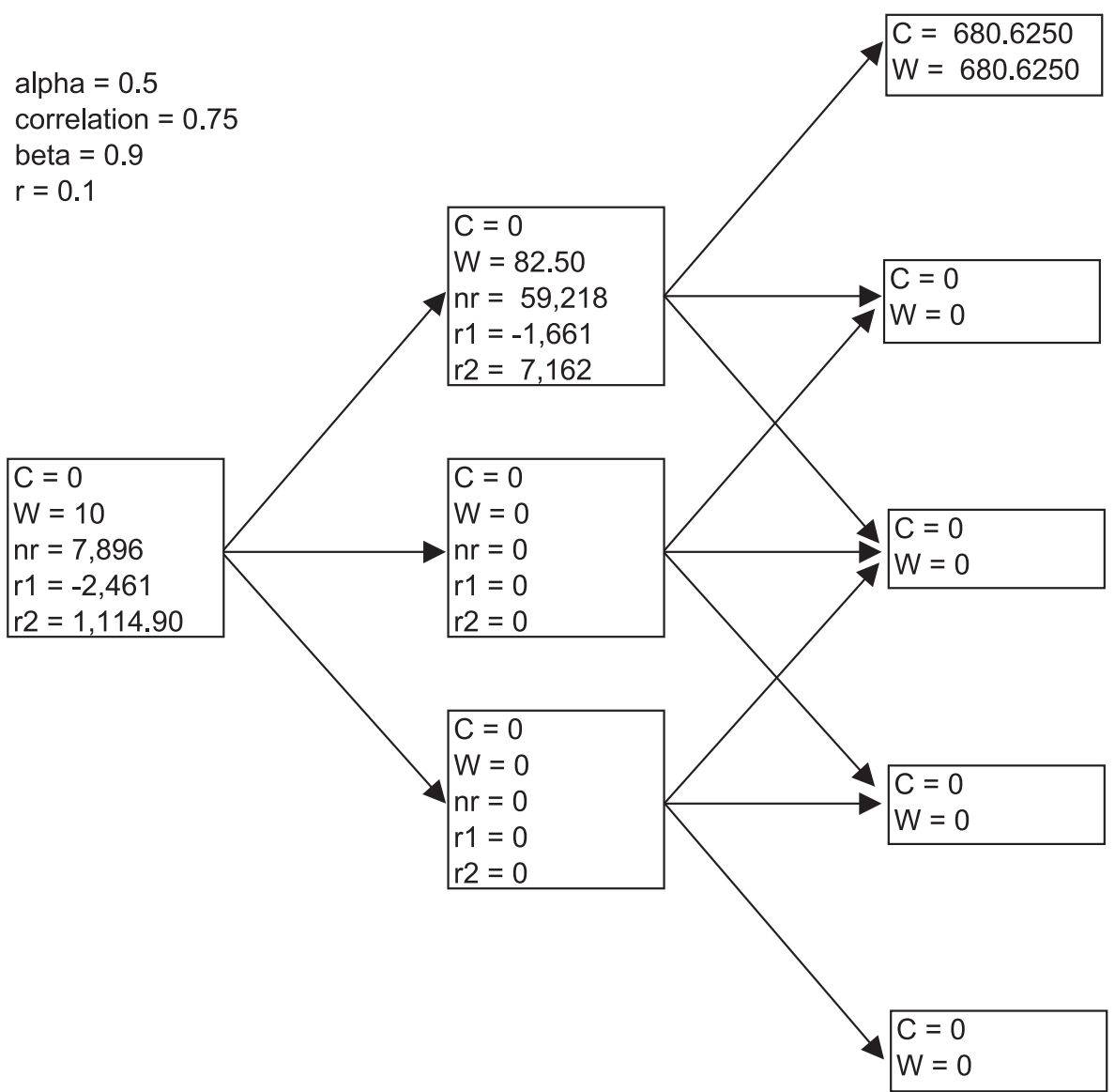

FIGURE 5. Consumption and investment strategies using Wang's distortion operator.

To see the impact of the loading parameter $\alpha$ on the consumption stream, Figure (6) plots the optimal consumption for different values of $\alpha$.

Around the value $\alpha=-0.8$, there is a switch in consumption from the lowest state, where it is nonzero and null elsewhere, to the highest state.

A closer look at the consumption process around $\alpha=-0.8$, is represented in Figure (7). This figure shows that in the transitory passage across the level $\alpha=0.8$, the intermediate consumption becomes nonzero.

From the examples above, it is clear that the linearity of the dual utility in consumption results in a corner solution in the optimisation problem. This is not a desirable feature in portfolio selection, although, as shown in the example, with 3 assets, diversification is possible. On the other hand, within the expected utility framework, a risk averse investor is always diversifying provided that the expected return of the risky asset is positive. 


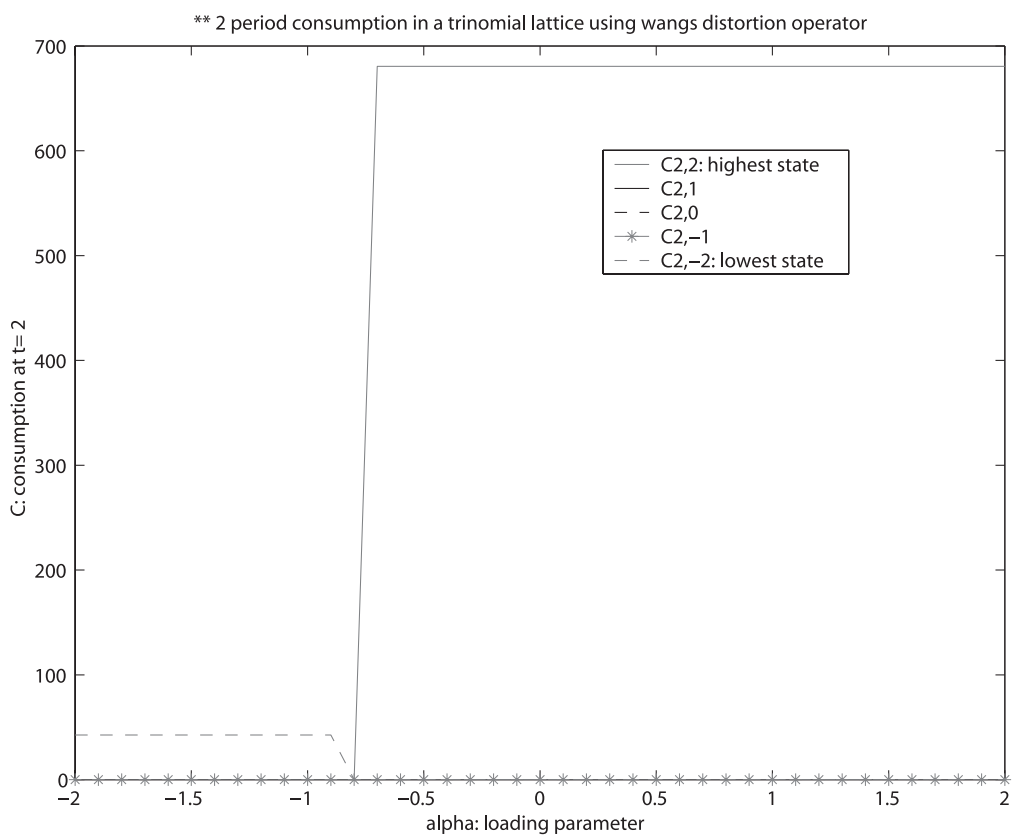

FIGURE 6. Consumption process as function of the loading parameter $\alpha$.

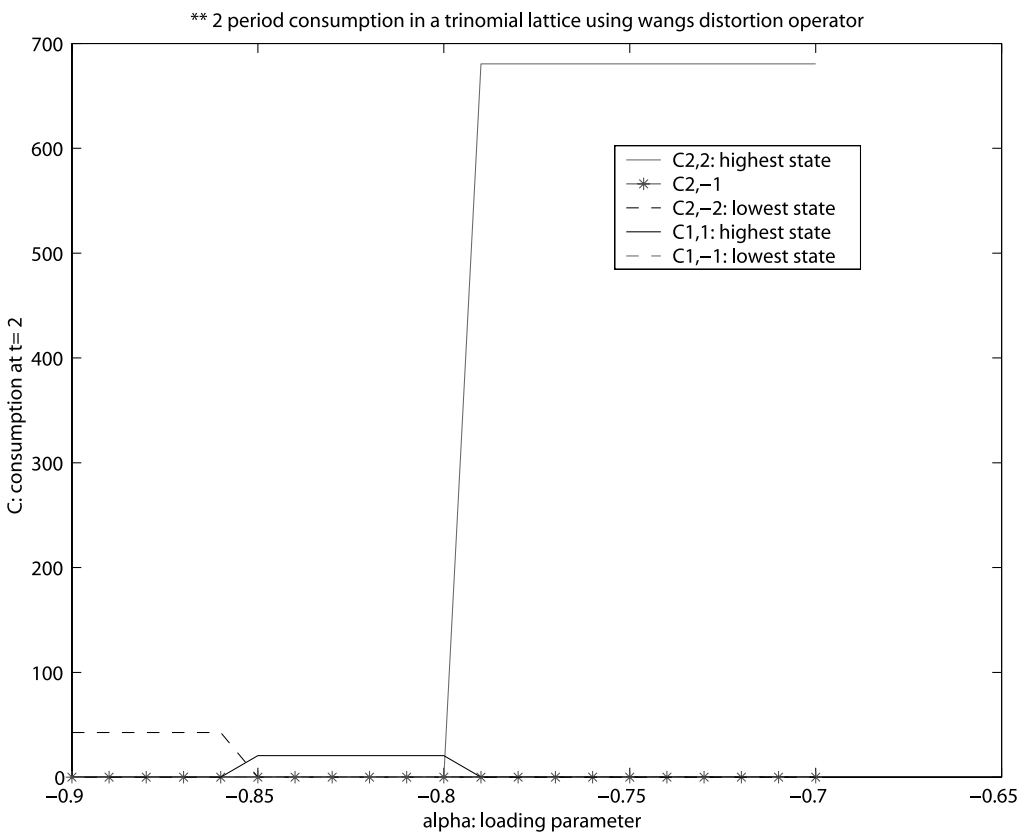

FIGURE 7. Consumption process as function of the loading parameter $\alpha$ (a zoom into -0.8 ) 

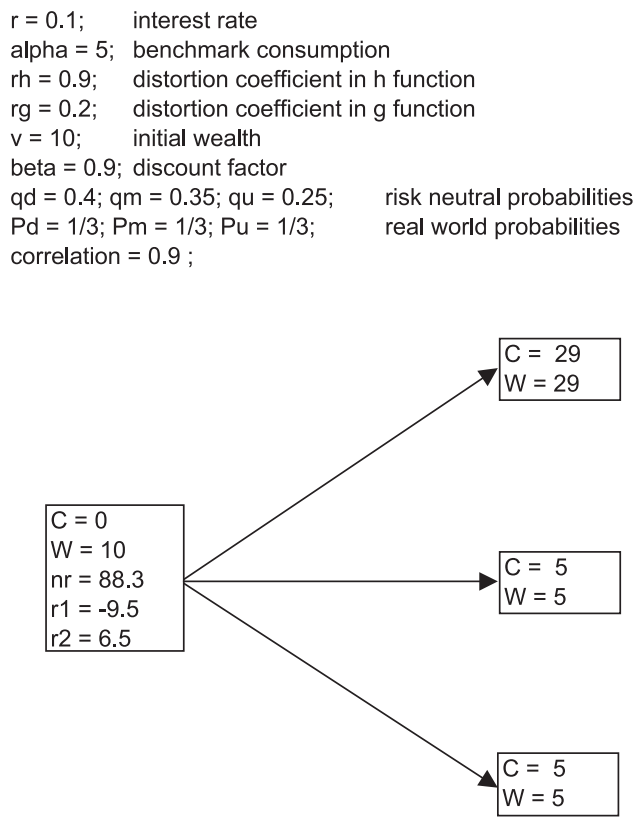

FIGURE 8. Optimal consumption and trading strategies using PH risk measure.

The second example is a numerical solution to the problem (??) where the risk measure is the one introduced by van der Hoek and Sherris. Figure (8) shows the optimal consumption and trading strategies for the parameters values indicated in the figure.

This numerical example shows that consumption at the end of the investment period is positive in all the states. This is due to the asymmetry resulting from pricing the downside of the risk using the distortion function $g(x)=x^{0.2}$ and the upside of the risk using $h(x)=1-(1-x)^{0.9}$. It is worth noting that the consumption in the middle and the down state equals the benchmark consumption $\alpha=5$. This is consequence of the linearity in Problem (??) where $C=\alpha$ is a corner solution.

\section{CONClusion}

In this paper we have provided a formal treatment of risk measures based on distortion functions in discrete-time setting. We have also shown that the risk neutral computational approach is well adapted to portfolio optimisation with such measures that don't lie within the expected utility framework.

The application to two different distortion operators shows that the portfolio consumption and investment rules are different from the expected utility results since the optimisation leads to corner solutions resulting from the linearity of 
the objective in the control variables. This is an undesirable feature and an important area that needs to be addressed before these non-expected utility risk measures can be confidently applied to asset allocation. This is an area for future research. One possibility is to consider combining expected and nonexpected utility measures as in Quiggin (1982 [15]).

\section{REFERENCES}

[1] Allais, M. (1953) Le comportement de l'homme rationnel devant le risque. Economertica, 21, 503-546.

[2] Bufman, G. and Leiderman, L. (1990) Consumption and asset returns under non-expected utility, some new evidence. Economics letters, 34, 231-235.

[3] CAmerer, C.F. (1989) An experimental test of several generalized utility theories. Journal of Risk and Uncertainty, 2(1), 61-104.

[4] Epstein, L.G. and Zin, S.E. (1989) Substitution, risk aversion, and the temporal behavior of consumption and asset returns: An empirical analysis. Carnegie-Mellon University, Pittersburg, PA.

[5] Epstein, L.G. and ZiN, S.E. (1989) Substitution, risk aversion, and the temporal behavior of consumption and asset returns: A theoretical framework. Econometrica, 57(4), 937-69.

[6] Fishburn, P.C. (1988) Nonlinear preference and utility theory. Johns Hopkins Series in the Mathematical Sciences, no 5. Baltimore and London, pp. xiv, 259.

[7] Giovannini, A. and Jorion, P. (1989) Time-series tests of a non-expected utility model of asset pricing. Columbia University, New York.

[8] Hamada, M. and Sherris, M. (2001) On the relationship between risk neutral valuation and pricing using distortion operators. Working paper.

[9] HE, H. (1990) Convergence from discrete to continuous-time contingent claims prices. The Review of Financial Studies, 3(4), 523-546.

[10] Ingersoll, J.E. (1987) "Theory of Financial Decision Making". Totowa, N.J. : Rowman and Littlefield.

[11] Kahneman, D. and Tversky, A. (1979) Prospect theory: An analysis of decision under risk. Econometrica, 47, 263-291.

[12] Koskievic, J.-M. (1999) An intertemporal consumption-leisure model with non-expected utility. Economics Letters, 64, 285-289.

[13] Kreps, D.M. and Porteus, E.L. (1978) Temporal resolution of uncertainty and dynamic choice theory. Econometrica, 46(1), 185-200.

[14] Osborne, M. (2001) "Simplicial Algorithms for Minimizing Polyhedral Functions". Cambridge University Press.

[15] Quiggin, J. (1982) A theory of anticipated utility. Journal of Economic Behavior and Organization, 3, 323-343.

[16] Roel, A. (1985) Risk aversion in quiggin and yaari's rank-order model of choice under uncertainty. The Economic Journal.

[17] Ross, S.A. (1981) Some stronger measures of risk aversion in the small and in the large with aplications. Econometrica, 49, 621-638.

[18] VAN DER Hoek, J. and SHerris, M. (2001) A class of non-expected utility risk measures and implications for asset allocations. Insurance: Mathematics and Economics 28, 69-82.

[19] WANG, S. (1996) Ambiguity aversion and the economics of insurance. University of Waterloo, research report 96-04.

[20] Wang, S. (1996) Premium calculation by transforming the layer premium density. Astin Bulletin, 26, 71-92.

[21] WANG, S. (1998) Implementation of proportional hazards transforms in ratemaking. Proceedings of the Casualty Actuarial Society LXXXV. Available for download on http://www. casact.org/pubs/proceed/proceed98/index.htm.

[22] WANG, S.S. (2000) A class of distortion operators for pricing financial and insurance risks. The Journal of Risk and Insurance, 36(1), 15-36. 
[23] YAARI, M.E. (1986) "Univariate and Multivariate Comparison of Risk Aversion: A New Approach". Cambridge University Press.

[24] YAARI, M.E. (1987) The dual theory of choice under risk. Econometrica, 55, 95-115.

MAHMOUd HAMADA

School of Finance and Economics

University of Technology of Sydney

Sydney, Australia

E-mail:mahmoud.hamada@uts.edu.au

APPENDiX A.

Properties of the Certainty Equivalent in Dual Theory

\section{A.1. General Case}

Let $g$ be a continuous, non-decreasing function, $g:[0,1] \rightarrow[0,1]$ with $g(0)=0$ and $g(1)=1$, and $X$ be a positive random variable representing a risk. The risk $X$ is measured by its certainty equivalent defined as:

$$
\Pi(X)=\int_{0}^{\infty} g\left(S_{X}(t)\right) d t
$$

In the discrete-time case, $X$ takes $n$ possible values $\left(X\left(\omega_{1}\right), X\left(\omega_{2}\right), \ldots, X\left(\omega_{n}\right)\right)$ with probabilities $\left(p_{1}, p_{2}, \ldots, p_{n}\right)$, where $n \in \mathbb{N} \backslash\{0\}$. In probabilistic notation, let $\Omega=\left\{\omega_{1}, \omega_{2}, \ldots, \omega_{n}\right\}$ be the probability space where $\omega_{i}, i \in\{1, \ldots, n\}$ are the states of the world, then $P\left[\omega_{i}\right]=p_{i} \forall i \in\{1, \ldots, n\}$.

This is typically the case of a tree model, where the number of states grows as time evolves. The following theorem gives an expression of $\Pi(X)$ in the discrete case.

Theorem 1 (Certainty equivalent). If $X$ is a random variable taking on $n$ distinct values $X\left(\omega_{1}\right), X\left(\omega_{2}\right), \ldots, X\left(\omega_{n}\right)$ with respective probabilities $p_{1}, p_{2}, \ldots, p_{n}$ then,

$$
\Pi(X)=\hat{\mathbb{E}}_{g}[X]
$$

where $\hat{\mathbb{E}}_{g}[X]$ is a weighted average of possible values of $X$, such that the weight $P^{g}\left(\omega_{i}\right)$ assigned to $X\left(\omega_{i}\right)$ is given by:

$$
P^{g}\left(\omega_{i}\right)=g\left(P\left[X \geq X\left(\omega_{i}\right)\right]\right)-g\left(P\left[X>X\left(\omega_{i}\right)\right]\right) \forall \omega_{i} \in \Omega
$$

$\hat{\mathbb{E}}_{g}[X]$ can be interpreted as an expectation where the probability assigned to a possible value of $X$ depends also on the other values. 
Proof. To simplify notation, let $x_{i}=X\left(\omega_{i}\right), i \in\{1, \ldots, n\}$. If we denote $x_{(i)}$ the ith value in increasing order (order statistics), then we have:

$$
\operatorname{Pr}[X>t]= \begin{cases}1 & \text { if } x_{(1)}>t \\ \sum_{k=2}^{n} p_{k} & \text { if } x_{(2)}>t \geq x_{(1)} \\ \sum_{k=3}^{n} p_{k} & \text { if } x_{(3)}>t \geq x_{(2)} \\ \cdots & \cdots \\ p_{n} & \text { if } x_{(n)}>t \geq x_{(n-1)} \\ 0 & \text { if } t \geq x_{(n)}\end{cases}
$$

Therefore,

$$
\begin{aligned}
\Pi(X) & =\int_{0}^{x_{(1)}} g(1) d t+\int_{x_{(1)}}^{x_{(2)}} g\left(\sum_{k=2}^{n} p_{k}\right) d t+\int_{x_{(2)}}^{x_{(3)}} g\left(\sum_{k=3}^{n} p_{k}\right) d t+\ldots+\int_{x_{(n-1)}}^{x_{(n)}} g\left(p_{n}\right) d t \\
& =x_{(1)}+\sum_{i=1}^{n-1} g_{i}^{p} \cdot\left[x_{(i+1)}-x_{(i)}\right] \\
& =\sum_{i=1}^{n}\left[g_{i-1}^{p}-g_{i}^{p}\right] x_{(i)}
\end{aligned}
$$

where

$$
\begin{aligned}
g_{i}^{p} & =g\left(\sum_{k=i+1}^{n} p_{k}\right) \\
& =g\left(P\left[X>x_{(i)}\right]\right)
\end{aligned}
$$

Since $g$ is an increasing function from [0,1] to [0,1], and $\forall i \sum_{k=i}^{n} p_{k}>\sum_{k=i+1}^{n} p_{k}$ then:

$$
\text { for all } i \text { in }\{1 . . n\}, P_{i}^{g} \equiv g\left(\sum_{k=i}^{n} p_{k}\right)-g\left(\sum_{k=i+1}^{n} p_{k}\right) \in[0,1]
$$

Moreover, $\sum_{i=1}^{n} P_{i}^{g}=g\left(\sum_{k=1}^{n} p_{k}\right)-g(0)=g(1)=1$. Therefore $\left\{P_{1}^{g}, P_{2}^{g}, \ldots, P_{n}^{g}\right\}$ define a probability measure on the probability space $\Omega=\left\{\omega_{1}, \omega_{2}, \ldots, \omega_{n}\right\}$.

This theorem states that in the discrete-time case, the certainty equivalent of $X$ is equivalent to an expectation under another probability measure. This has been shown when all the possible values of $X$ are distinct. The question that arises immediately is: what happens in the case when some possible values of $X$ coincide? The following example provides an insight into this question. 
In the general case when some values of $X$ coincide, order them in increasing order, then from each set of equal values keep only one value and assign the probability of the set to this value. Thus, a new variable $Y$ is defined in such a way that all the elements of $Y$ are strictly increasing with adjusted probability weights such that the identity $\Pi(X)=\Pi(Y)$ is satisfied.

Another approach consists of keeping the redundant values and dividing the probability weights by the number of these values. This is the idea of the next corollary:

Corollary 1. If $X$ is a random variable taking $n$ possible values $X\left(\omega_{1}\right), X\left(\omega_{2}\right), \ldots$, $X\left(\omega_{n}\right)$ with probabilities $\left(p_{1}, p_{2}, \ldots, p_{n}\right)$, then,

$$
\Pi(X)=\tilde{\mathbb{E}}_{g}[X]
$$

where $\tilde{\mathbb{E}}_{g}[X]$ is a weighted average of possible values of $X$, such that the weight $\tilde{P}^{g}$ assigned to $X\left(\omega_{i}\right)$ is given by:

$$
\tilde{P}^{g}\left(\omega_{i}\right)=\frac{g\left(P\left[X \geq X\left(\omega_{i}\right)\right]\right)-g\left(P\left[X>X\left(\omega_{i}\right)\right]\right)}{\#\left\{X\left(\omega_{j}\right): X\left(\omega_{j}\right)=X\left(\omega_{i}\right)\right\}} \text { for all } \omega_{i} \text { in } \Omega
$$

where the notation $\#\left\{X\left(\omega_{j}\right): X\left(\omega_{j}\right)=X\left(\omega_{i}\right)\right\}$ stands for the number of values $X\left(\omega_{j}\right)$ equal to $X\left(\omega_{i}\right)$.

Proof. The idea of the proof is given in the previous example. In formal terms, let

$$
\Psi_{s}=\left\{i \in\{1, \ldots, n\}: X\left(\omega_{i}\right)=X\left(\omega_{s}\right)\right\}, s \in\{1, \ldots, n\}
$$

and

$$
P^{g}\left(\omega_{i}\right)=g\left(P\left[X \geq X\left(\omega_{i}\right)\right]\right)-g\left(P\left[X>X\left(\omega_{i}\right)\right]\right) \forall \omega_{i} \in \Omega
$$

We have: $\forall i \in \Psi_{s}, P^{g}\left(\omega_{i}\right)=P^{g}\left(\omega_{s}\right)$ and so

$$
\sum_{i \in \Psi_{s}} P^{g}\left(\omega_{i}\right) X\left(\omega_{i}\right)=\left|\Psi_{s}\right| P^{g}\left(\omega_{s}\right) X\left(\omega_{s}\right)
$$

where

$$
\left|\Psi_{s}\right|=\#\left\{X\left(\omega_{j}\right): X\left(\omega_{j}\right)=X\left(\omega_{s}\right)\right\}
$$

Define a new random variable $Y$ and a subset of indices $S \subseteq\{1, \ldots, n\}$, such that: 


$$
\begin{aligned}
Y\left(\omega_{i}\right) & =X\left(\omega_{i}\right) \quad \forall i \in S \\
Y\left(\omega_{i}\right) & \neq Y\left(\omega_{j}\right) \text { for } i \neq j, \forall(i, j) \in S^{2} \\
\operatorname{Image}(Y) & =\operatorname{Image}(X)
\end{aligned}
$$

where Image $(X)$ is the set of all possible values taken by $X$.

By applying the theorem to $Y$ whose values are all distinct, we have:

$$
\begin{aligned}
\Pi(Y)= & \sum_{s \in S} P^{g}\left(\omega_{s}\right) Y\left(\omega_{s}\right) \\
& \sum_{s \in S} \frac{\left|\Psi_{s}\right| P^{g}\left(\omega_{s}\right) X\left(\omega_{s}\right)}{\left|\Psi_{s}\right|} \\
= & \sum_{s \in S} \frac{1}{\left|\Psi_{s}\right|} \sum_{i \in \Psi_{s}} P^{g}\left(\omega_{i}\right) X\left(\omega_{i}\right) \\
= & \sum_{i=1}^{n} \frac{P^{g}\left(\omega_{i}\right) X\left(\omega_{i}\right)}{\#\left\{X\left(\omega_{j}\right): X\left(\omega_{j}\right)=X\left(\omega_{i}\right)\right\}}
\end{aligned}
$$

Since

$$
\operatorname{Pr}[X>t]=\operatorname{Pr}[Y>t] \forall t \geq 0
$$

then,

$$
\Pi(Y)=\Pi(X)
$$

\section{A.2. Application: Van der Hoek and Sherris class of risk measures}

Corollary 2 (Position of $\alpha$ ). If $X$ is a multinomial discrete random variable taking the values $\left(x_{1}, x_{2}, \ldots, x_{n}\right)$ such that $x_{1}<x 2<\ldots<x_{n}$, with probabilities $\left(p_{1}, p_{2}, \ldots\right.$, $\left.p_{n}\right)$, then,

- If $\alpha \leq x_{1}$, then:

$$
\begin{aligned}
H(X) & =x_{1}+\sum_{i=1}^{n-1} h_{i}^{p}\left[x_{i+1}-x_{i}\right] \\
& =\sum_{i=1}^{n}\left[h_{i-1}^{p}-h_{i}^{p}\right] x_{i}
\end{aligned}
$$


- If $\alpha \geq x_{n}$, then:

$$
\begin{aligned}
H(X) & =x_{n}+\sum_{i=1}^{n-1} g_{i}^{p}\left[x_{i}-x_{i+1}\right] \\
& =\sum_{i=1}^{n}\left[g_{i}^{p}-g_{i-1}^{p}\right] x_{i}
\end{aligned}
$$

- If $\alpha \in\left[x_{r}, x_{r+1}\right)$ where $r \in\{1, \ldots, n-1\}$, then:

$$
H(X)=\left[1-h_{r}^{p}-g_{r}^{p}\right] \alpha+\sum_{i=1}^{r}\left[g_{i}^{p}-g_{i-1}^{p}\right] x_{i}+\sum_{i=r+1}^{n}\left[h_{i-1}^{p}-h_{i}^{p}\right] x_{i}
$$

where

$$
h_{i}^{p}=h\left(1-\sum_{k=1}^{i} p_{k}\right) \text { and } g_{i}^{p}=g\left(\sum_{k=1}^{i} p_{k}\right)
$$

This corollary illustrates the idea of pricing the upside and the downside of the risk differently. In effect, for outcomes $x_{i}$ 's below the level $\alpha$, the probability distribution is distorted by the function $g$, and for outcomes $x_{i}$ 's above the level $\alpha$, the probability distribution is distorted by the function $h$. This is a flexible way to price risk around some benchmark $\alpha$. The choice of the distortion functions $g$ and $h$ reflects the risk behaviour of the investor. Indeed, $h$ is convex, then $2 h(x) \leq h(x-1)+h(x+1)$ wherever $h$ is defined. Therefore

$$
h\left(1-\sum_{k=1}^{i-1} p_{k}\right)-h\left(1-\sum_{k=1}^{i} p_{k}\right)<h\left(1-\sum_{k=1}^{i-2} p_{k}\right)-h\left(1-\sum_{k=1}^{i-1} p_{k}\right)
$$

or

$$
h_{i-1}^{p}-h_{i}^{p}<h_{i-2}^{p}-h_{i-1}^{p}
$$

So the probability assigned to the outcome $x_{i}$ is less than the probability assigned to $x_{i-1}$. In other terms, the investor assigns lower probabilities to higher outcomes. The more risk averse the investor, the more convex the distortion function $h$. The same argument applies to the concavity of $g$.

Furthermore, the choice of $h$ with respect to $g$ reflects how the investor considers the risk with respect to the benchmark $\alpha$. For some choice of $g$ and $h$, pricing risk does not depend on $\alpha$. This is the idea of the following proposition: 
Proposition 2 (Conjugate). Let $X$ be a random variable taking on $n$ distinct values $X\left(\omega_{1}\right), X\left(\omega_{2}\right), \ldots, X\left(\omega_{n}\right)$ with probabilities $p_{1}, p_{2}, \ldots, p_{n}$. In the case when $h$ and $g$ are conjugate, we have

$$
H(X)=\hat{\mathbb{E}}_{h}[X]
$$

where the weighted average weights are given by

$$
\mathbf{P}^{h}(\omega)=h(\mathbf{P}[X \geq X(\omega)])-h(\mathbf{P}[X>X(\omega)]) \forall \omega \in \Omega
$$

Proof. In the case when $h$ and $g$ are conjugate, i.e., $h(1-x)=1-g(x)$, in Appendix 2, we show that:

$$
H(X)=H_{0,0, h}(X)=\int_{0}^{\infty} h(P[X>t]) d t
$$

Therefore, the proposition follows from Theorem (Certainty equivalent).

Proposition 3 (No order assumption). For a multinomial discrete random variable $X$ taking the values $\left(x_{1}, x_{2}, \ldots, x_{n}\right)$, with probabilities $\left(p_{1}, p_{2}, \ldots, p_{n}\right), x_{i} \neq x_{j}$ if $i \neq j$ then,

$$
H(X)=\text { Const }+\sum_{i=1}^{n}\left(g_{i}^{p, X} I_{\left\{x_{i} \leq \alpha\right\}}+h_{i}^{p, X} I_{\left\{x_{i}>\alpha\right\}}\right) x_{i}
$$

where

$$
\begin{aligned}
& g_{i}^{p, X}=g\left(\sum_{\left\{x_{k} \leq x_{i}\right\}} p_{k}\right)-g\left(\sum_{\left\{x_{k}<x_{i}\right\}} p_{k}\right) \\
& h_{i}^{p, X}=\left[h\left(1-\sum_{\left\{x_{k}<x_{i}\right\}} p_{k}\right)-h\left(1-\sum_{\left\{x_{k} \leq x_{i}\right\}} p_{k}\right)\right] \\
& \text { Const }=\left[1-h\left(1-\sum_{\left\{x_{k} \leq \alpha\right\}} p_{k}\right)-g\left(\sum_{\left\{x_{k} \leq \alpha\right\}} p_{k}\right)\right] \alpha
\end{aligned}
$$

and the notation

$$
\sum_{\left\{x_{k} \leq \alpha\right\}} p_{k}=\sum_{k=1}^{n} p_{k} I_{\left\{x_{k} \leq \alpha\right\}}
$$

where $I_{\left\{x_{k} \leq \alpha\right\}}$ is the indicator function on the set $\left\{x_{k} \leq \alpha\right\}$. 
APPENDIX B.

Proof of Proposition (Order ASSUMPTION)

Proof. For $x_{1}<x_{2}<\ldots<x_{n}$ we have:

$$
\left(x_{1}-\alpha\right)^{+} \leq\left(x_{2}-\alpha\right)^{+} \leq \ldots \leq\left(x_{n}-\alpha\right)^{+} \text {and }\left(\alpha-x_{1}\right)^{+} \geq\left(\alpha-x_{2}\right)^{+} \geq \ldots \geq\left(\alpha-x_{n}\right)^{+} \text {, so }
$$

$$
\operatorname{Pr}\left[(X-\alpha)^{+}>t\right]= \begin{cases}1 & \text { if }\left(x_{1}-\alpha\right)^{+}>t \\ \sum_{k=2}^{n} p_{k} & \text { if }\left(x_{2}-\alpha\right)^{+}>t \geq\left(x_{1}-\alpha\right)^{+} \\ \sum_{k=3}^{n} p_{k} & \text { if }\left(x_{3}-\alpha\right)^{+}>t \geq\left(x_{2}-\alpha\right)^{+} \\ \cdots & \cdots \\ p_{n} & \text { if }\left(x_{n}-\alpha\right)^{+}>t \geq\left(x_{n-1}-\alpha\right)^{+} \\ 0 & \text { if } t \geq\left(x_{n}-\alpha\right)^{+}\end{cases}
$$

and

$$
\operatorname{Pr}\left[(\alpha-X)^{+}>t\right]= \begin{cases}1 & \text { if }\left(\alpha-x_{n}\right)^{+}>t \\ \sum_{k=1}^{n-1} p_{k} & \text { if }\left(\alpha-x_{n-1}\right)^{+}>t \geq\left(\alpha-x_{n}\right)^{+} \\ \sum_{k=1}^{n-2} p_{k} & \text { if }\left(\alpha-x_{n-2}\right)^{+}>t \geq\left(\alpha-x_{n-1}\right)^{+} \\ \cdots & \cdots \\ p_{1} & \text { if }\left(\alpha-x_{1}\right)^{+}>t \geq\left(\alpha-x_{2}\right)^{+} \\ 0 & \text { if } t \geq\left(\alpha-x_{1}\right)^{+}\end{cases}
$$

Therefore,

$$
\begin{aligned}
& H(X)=\alpha+\int_{0}^{\left(x_{1}-\alpha\right)^{+}} h(1) d t+\int_{\left(x_{1}-\alpha\right)^{+}}^{\left(x_{2}-\alpha\right)^{+}} h\left(\sum_{k=2}^{n} p_{k}\right) d t+ \\
& \int_{\left(x_{2}-\alpha\right)^{+}}^{\left(x_{3}-\alpha\right)^{+}} h\left(\sum_{k=3}^{n} p_{k}\right) d t+\ldots+\int_{\left(x_{n-1}-\alpha\right)^{+}}^{\left(x_{n}-\alpha\right)^{+}} h\left(p_{n}\right) d t-\int_{0}^{\left(x_{n}-\alpha\right)^{+}} g(1) d t- \\
& \int_{\left(\alpha-x_{n}\right)^{+}}^{\left(\alpha-x_{n-1}\right)^{+}} g\left(\sum_{k=1}^{n-1} p_{k}\right) d t-\int_{\left(\alpha-x_{n-1}\right)^{+}}^{\left(\alpha-x_{n-2}\right)^{+}} g\left(\sum_{k=1}^{n-2} p_{k}\right) d t-\ldots-\int_{\left(\alpha-x_{2}\right)^{+}}^{\left(\alpha-x_{1}\right)^{+}} g\left(p_{1}\right) d t
\end{aligned}
$$

By using

$$
\int_{\left(x_{i}-\alpha\right)^{+}}^{\left(x_{i+1}-\alpha\right)^{+}} h\left(\sum_{k=i+1}^{n} p_{k}\right) d t=h\left(1-\sum_{k=1}^{i} p_{k}\right)\left[\left(x_{i+1}-\alpha\right)^{+}-\left(x_{i}-\alpha\right)^{+}\right]
$$


and

$$
\int_{\left(\alpha-x_{i+1}\right)^{+}}^{\left(\alpha-x_{i}\right)^{+}} g\left(\sum_{k=1}^{i} p_{k}\right) d t=-g\left(\sum_{k=1}^{i} p_{k}\right)\left[\left(\alpha-x_{i+1}\right)^{+}-\left(\alpha-x_{i}\right)^{+}\right]
$$

we get the desired result.

APPENDIX C.

THe CASE WHEN $G$ AND $H$ ARE CONJUGATE

We propose to show that

$$
H(X)=H_{0,0, h}(X)=\int_{0}^{\infty} h(P[X>t]) d t
$$

First, it is easy to check that, for $t \geq 0$,

$$
\begin{gathered}
S_{(X-\alpha)^{+}}(t)=S_{X}(\alpha+t) \\
S_{(\alpha-X)^{+}}(t)=1-S_{X}(\alpha-t)
\end{gathered}
$$

and since $h$ and $g$ are conjugate, we have:

$$
\begin{gathered}
h^{\prime}(x)=g^{\prime}(1-x) \\
H(X)=\alpha+\int_{0}^{\infty} h\left(S_{(X-\alpha)^{+}}(t)\right) \cdot d t-\int_{0}^{\infty} g\left(S_{(\alpha-X)^{+}}(t)\right) \cdot d t \\
=\alpha+\int_{0}^{\infty} h\left(S_{X}(\alpha+t)\right) \cdot d t+\int_{0}^{\infty} g\left(1-S_{X}(\alpha-t)\right) \cdot d t \\
=\alpha-\int_{0}^{\infty} t h^{\prime}\left(S_{X}(\alpha+t)\right) \cdot d S_{X}(\alpha+t)-\int_{0}^{\infty} t g^{\prime}\left(1-S_{X}(\alpha-t)\right) \cdot d S_{X}(\alpha-t) \\
=\alpha-\int_{\alpha}^{\infty}(u-\alpha) h^{\prime}\left(S_{X}(u)\right) \cdot d S_{X}(u)-\int_{\alpha}^{-\infty}(\alpha-v) g^{\prime}\left(1-S_{X}(v)\right) \cdot d S_{X}(v) \\
=\alpha+\alpha \int_{\alpha}^{\infty} h^{\prime}\left(S_{X}(u)\right) \cdot d S_{X}(u)-\int_{\alpha}^{\infty} u h^{\prime}\left(S_{X}(u)\right) \cdot d S_{X}(u) \\
-\alpha \int_{\alpha}^{-\infty} h^{\prime}\left(S_{X}(v)\right) \cdot d S_{X}(v)+\int_{\alpha}^{-\infty} v h^{\prime}\left(S_{X}(v)\right) \cdot d S_{X}(v)
\end{gathered}
$$




$$
\begin{aligned}
& =\alpha+\alpha \int_{-\infty}^{\infty} h^{\prime}\left(S_{X}(u)\right) \cdot d S_{X}(u)-\int_{-\infty}^{\infty} v h^{\prime}\left(S_{X}(v)\right) \cdot d S_{X}(v) \\
& =\alpha-\alpha \underbrace{\int_{-\infty}^{\infty} h^{\prime}\left(S_{X}(u)\right) \cdot d F_{X}(u)}_{=1}-\int_{-\infty}^{\infty} v h^{\prime}\left(S_{X}(v)\right) \cdot d S_{X}(v) \\
& =\int_{-\infty}^{\infty} v h^{\prime}\left(S_{X}(v)\right) \cdot d F_{X}(v) \\
& =\int_{0}^{\infty} h\left(S_{X}(v)\right) \cdot d v
\end{aligned}
$$

